\title{
HIGH-ORDER SIMULATION OF THE FLOW INSTABILITY OVER A NON-CIRCULAR STRUCTURE
}

\author{
Matthieu Minguez \\ Seal Engineering \\ (Technip Subsidiary) \\ Nimes, France
}

\author{
Philippe Meliga \\ M2P2, CNRS \\ (Université d'Aix Marseille) \\ Marseille, France
}

\author{
Eric Serre \\ M2P2, CNRS \\ (Université d'Aix Marseille) \\ Marseille, France
}

\section{ABSTRACT}

Bluff bodies can be prone to well-known self-excited instability such as galloping. Opposite to classical and well documented Vortex Induced Vibrations (VIV), oscillation amplitude is not bounded in such a way galloping-like instability can alter the integrity of the structure on short term. Although stability criteria exist in the literature [1], there is a real lack considering the cause and the way to inhibit such instability.

It is consequently proposed in this paper to investigate the flow over the classical 'non-circular' structure, i.e the square box body, by means of advanced Spectral Large Eddy Simulation (LES) solver, [16], [17] \& [18]. The main objectives of this numerical analysis will be to provide an accurate solution to underline the possible mechanisms that trigger the instability as well as a base solution for future wake stability \& control analysis, [6].

\section{INTRODUCTION}

Bundles arrangements are current solutions for riser towers or oil export lines. Most of the time, they are characterized by the loss of symmetry of revolution. When plunged into strong current, they can be prone to well-known fluid structure kind of interactions that can be either Vortex Induced Vibrations (VIV) or galloping-like instability. In the case of VIV (e.g [10], [11],
[12], [13]), the 'self-limited' oscillation amplitudes result from a lock-in phenomenon between the flow and the structure dynamics and for, which damping and non-linearity's play a stabilizing role. Conversely, oscillations in galloping-like instability are no more 'self-limited' but so-called 'self-excited' (e.g [1], [20]), which lies in the occurrence of negative damping [1], the hydrodynamic forces caused by small relative motions of the structure acting to amplify these motions, which results in growing amplitudes only limited by nonlinear effects. If VIV analysis is now currently integrated into the design process of sub-sea structure as possible source of Fatigue, the risk for occurrence of galloping is not as well assessed.

Experimental studies have been performed in the past to highlight and characterize such instability. A majority of them are related to aerodynamic issues, e.g [20], [21], [22], but few focus on the hydrodynamic component of the problem, e.g [1], [2], [12]. Blevins was the first author to really differentiate aerodynamic 'fluttering' from hydrodynamic galloping. In [1], he established a predictive linear criterion for the occurrence of the galloping, which still remains a reference and is invoked most often at a basis for numerous galloping analyses. In practice, it requires the knowledge of the variation of the hydrodynamic coefficients (drag \& lift) with regard to the flow incidence, which is most of the time achieved by means of Computational Fluid Dynamic (CFD) simulations or model tests, e.g [2], [12]. 
One of the classical study cases for the analysis of the galloping instability is the 'square cylinder'. In the last decades, several studies have especially provided exhaustive characterization of this bluff body response when plunged into current. However, recent experimental R\&D campaign, [2], has unravelled novel dynamical features with complex vortex interactions in the very near wake of the cylinder that remains misunderstood to date. Our objective here is to shed some light on the role played by these complex vortex interactions in the occurrence of the galloping instability.

For this purpose, CFD tool, once validated, appears as the perfect complement to experiments for complex flow features investigations. Despite the simplicity of the square cylinder geometry, the flow that develops is very complex, fully threedimensional and unsteady, including transition regions to turbulence as well as flow separations along the sidewall. Model after the flow past the circular cylinder, [23], the square cylinder flow is very challenging for numerical modelling. The different ERCOFTAC benchmarks ([24] \& [25]) at highReynolds $(R e=22000)$ demonstrate the interest of the numerical community for this configuration. They notably assessed the ability of RANS modelling to predict the main integral quantities (drag \& Strouhal) but also, their incapacity to render finer physics such as turbulence fluctuations.

In this paper, we propose to investigate the flow past the 'square cylinder' using an 'in-house' advanced CFD tool, i.e. spectral Large-Eddy Simulation (LES). The solver is now mature enough to be applied in such a complex configuration. By the past, previous works have (e.g. [16], [17], [18], [19]) underlined its ability to recover complex multi-scale interactions for which classical RANS approaches are not intrinsically adapted. We expect the use of this advanced modelling will offer comprehensive response elements on the main flow feature, as well as the source of galloping instability. This study coming within the framework of the special session on Verification \& Validation, [23], represents the first step of a more extensive collaborative research program based on the control of hydrodynamic instabilities, for which we believe the use of the most possible accurate numerical solutions is a crucial point.

\section{NOMENCLATURE}

$\begin{array}{ll}\text { CFD } & \begin{array}{l}\text { Computational Fluid Dynamic } \\ C_{y}\end{array} \\ D & \begin{array}{l}\text { Transverse Hydrodynamic coefficient } \\ \text { Bluff Body Characteristic dimension } \\ \text { (diameter) }\end{array} \\ \text { FIV } & \text { Flow Induced Vibration } \\ f_{\mathrm{n}} & \text { Natural frequency of the structure } \\ \text { FSI } & \text { Fluid Structure Interaction } \\ \text { ERCOFTAC } & \text { European Research Community On Flow, }\end{array}$

Turbulence and Combustion

$\begin{array}{ll}\text { KH } & \text { Kelvin Helmholtz } \\ \text { LES } & \text { Large-Eddy Simulation } \\ m_{a} & \text { Added Mass } \\ m_{N} & \text { SVV Activation parameter } \\ N & \text { Degree of polynomial approximation } \\ \text { NW } & \text { Near Wall Region } \\ Q_{N} & \text { SVV Kernel } \\ \text { RANS } & \text { Reynolds Averaged Navier Stokes } \\ \text { Re } & \text { Re number } \\ \text { St } & \text { Strouhal number } \\ \text { SVV } & \text { Spectral Vanishing Viscosity } \\ \text { SVV-LES } & \text { Large Eddy Simulation via SVV modelling } \\ \text { SVV-NW } & \text { SVV Wall Treatment } \\ \text { VIV } & \text { Vortex Induced Vibrations } \\ \text { VK } & \text { Von Karman } \\ \zeta & \text { Structural Damping } \\ \chi & \text { Bluff body characteristic function } \\ \chi_{B L} & \text { Boundary Layer characteristic function } \\ \varepsilon_{N} & \text { SVV Amplitude parameter } \\ v & \text { Kinetic viscosity } \\ \Delta_{S V V} & \end{array}$

\section{CURRENT GALLOPING INVESTIGATION WAY}

The main assumption in galloping analysis is that the fluid force is quasi-steady; i.e. the fluid force on the structure is exclusively determined by the instantaneous relative velocity and the angle of attack of the flow with regard to the structure. To ensure such assumption, the structure motion period should be consequently much larger than the main fluid characteristic time scale (refer to eq. (1) where $U$ is the free stream velocity and $f_{\mathrm{n}}$ the structure natural period).

$$
\text { (1) } \frac{U}{f_{n} \cdot D}>10
$$

Consequently, the galloping instability would preferentially mobilize the first natural mode of the structure. Based on such assumption, the galloping instability can be investigated by means of static model tests or numerical tool (CFD) by performing parametric analysis of the flow incidence.

Analytical developments made by Blevins, [1], describe plunge (translational) galloping instabilities of square cylinder subjected to steady flow with one degree of freedom. Based on 
linearised motion equations, Blevins establishes a criterion to evaluate the risk of occurrence of the instability solely accounting for the structure geometry and hydrodynamics (eq. (2)).

$$
\text { (2) } \frac{\partial C_{y}}{\partial \alpha}<0
$$

Namely, if the variation of the transverse hydrodynamic coefficient, $C_{y}$, with regard to the flow incidence, $\alpha$, is negative, a risk of galloping instability exists and should be investigated in more detailed. In this perspective, Blevins proposes a second development based on the intrinsic properties of the structure (inertia and damping, $\zeta$ ) to refine the previous criterion and determine the onset velocity of the instability Eq. (3)

$$
\text { (3) } \frac{4\left(m+m_{a}\right) \cdot(2 \pi \xi)}{\rho D^{2}} / \frac{U}{f D}+\frac{\partial C_{y}}{\partial \alpha}>0
$$

Traditionally, the galloping instability is investigated by either coupling the Blevins's criteria with CFD or model tests (refer to [12]), or by performing more sophisticated dynamical model tests as proposed in [1].

\section{BACKGROUND \& RESEARCH SCOPE OF WORK}

\section{High-order Turbulent Flow Solver}

When the smallest scales of the turbulence interact with the largest coherent structures of the flow up to trigger instability, it becomes more and more challenging to numerically recover the sharpness of the involved physic. The order of the numerical schemes and of the turbulence modelling should be consequently high enough to capture and to not dissipate the involved driven scales.

In this frame, it is proposed to resort to spectral approximation, well-known to achieve 'spectral' accuracy. That means, the error decays at a rate which solely depends on the smoothness of the solution. Spectral methods are consequently much less diffusive than lower order ones but are equally well known to difficulty compute high-Reynolds number flows. The accumulation of energy in the high spatial frequency range generally leads to a divergence of the computations. As a solution, a Spectral Vanishing Viscosity (SVV) technique has been proposed as an efficient stabilization method of highly accurate spectral approximation, [14]. Contrarily to many stabilization techniques that generally destroy the spectral accuracy of the algorithm, SVV possesses the property of preserving the spectral accuracy. The previous works have progressively demonstrated the ability to recover turbulent flow,
[15], [16], and more recently industrial high-Reynolds configurations, [17] \& [18].

The proposed work aims at improving the understanding of the flow instability that may trigger the galloping of the noncircular structure. In this perspective, spectral methods (i.e SVV-LES technique) are used to accurately capture the multiscale interactions that develop over the body.

\section{Wake Flow Stability \& Control}

Future work will especially feature control of the wake dynamics by means of imposed, local disturbances. Our focus will be on the effect of a secondary control cylinder on the shedding activity. A seminal analysis has been carried out by [9] on flow past a circular cylinder close to the instability threshold. These authors clearly evidenced that a suitably positioned control cylinder can either suppress the shedding itself, either or decrease the shedding frequency. They represented the influence of the control cylinder position in terms of sensitivity maps showing the regions around the primary cylinder in which the shedding activity was most affected. Both effects are highly valuable for the intended application, for instance aero/hydroelastic instabilities can be alleviated if the flow is stabilized, or undesirable vortex-induced vibrations can be avoided (e.g changing its natural). Several studies have also assessed the potential of this approach on a variety of flow configurations at practically meaning full Reynolds numbers of order $10^{4}-10^{6}$ ([8], [5], [3], [4], [7]). The general conclusion to be drawn is that varying the position of the control cylinder can increase or decrease the shedding frequency. As for the shedding itself, it can be alleviated but not extinguished on behalf of the large Reynolds numbers.

For the test case considered herein, we will first compute the sensitivity of the shedding activity from LES simulations embedding the presence of the control cylinder. All computations will be undertaken in the limit of small cylinder ratios, i.e., control cylinders much smaller than the main cylinder. The obtained results will then be compared to theoretical maps computed using the adjoin-based formalism of [6]. The latter uses the concept of structural sensitivity, which stems from the framework of stability analyses in laminar flows, and allows identifying the most sensitive regions beforehand, without ever having to calculate the actual controlled states. Our ambition is to demonstrate that the obtained structural sensitivity will indicates where and how to control the flow efficiently using small-amplitude actuation. In return, it should avoid resorting to a heuristic « trial \& error » procedures.

\section{NUMERICAL MODELLING}

The geometry corresponds to the one proposed in [25]. A square cylinder of height $\mathrm{D}$ is placed at the origin of the 
coordinate system within a computational domain of dimension $(-4.5 \mathrm{D} ; 15 \mathrm{D}) \times(-7 \mathrm{D} ; 7 \mathrm{D}) \times(-2 \mathrm{D} ; 2 \mathrm{D})$ respectively in the $(\mathrm{x} ; \mathrm{y}$; $\mathrm{z})$ directions, refer to Figure 1 . At the inlet, a uniform velocity profile $U_{\infty}$ is imposed. The characteristic Reynolds number of the flow, based on $U_{\infty}$ and $\mathrm{D}$, is equal to $\mathrm{Re}=22000$.

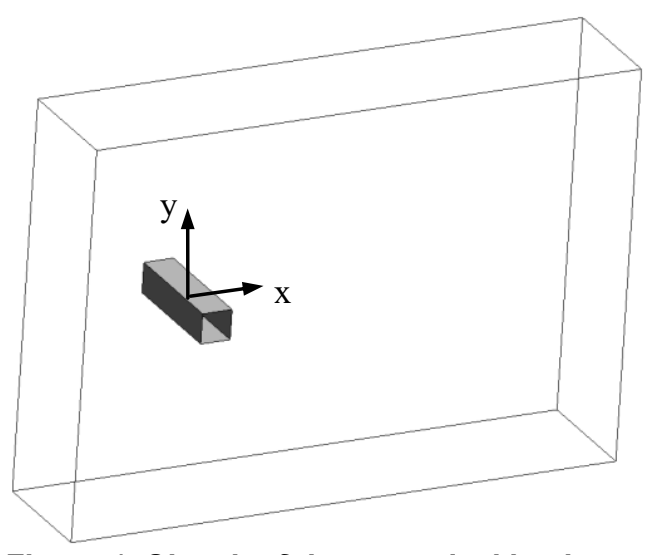

Figure 1: Sketch of the numerical basin

The numerical method is based on a multi-domain ChebyshevFourier approximation. In the stream wise $\mathrm{x}$-direction, the computational domain is decomposed in 8 non-overlapping subdomains of different lengths, depending on the flow region. In each sub-domain, a collocation Chebyshev method is used in the y-vertical and stream wise directions, whereas a FourierGalerkin method is used in the z-span wise periodic direction. Finally, each one of 8 sub-domains is discretized in $(41 \times 501 \times 128)$ nodes respectively in the direction $(x, y, z)$. In order to refine the Gauss-Lobatto-Chebyshev grid around the bluff body, a mapping is used to finally accumulate mesh-points at the bluff body, especially in y-direction. By such a way the first grid point is located at a distance of $\Delta y / D=0.1$ that should offer a relatively accurate description of the boundary layer, as already mentioned in the conclusions of the ERCOFTAC test case ([25]). The square cylinder is modelled using a pseudopenalization technique, see [16] for more details. The discretisation in time is based on a fractional step method globally $2^{\text {nd }}$ order accurate.

The LES is achieved by a Spectral Vanishing Viscosity (SVV) stabilization method, allowing a damping of the highest frequencies of the solution (see in [18] for details). The NavierStokes equations are completed with a supplementary diffusion term and may then be expressed with a SVV-modified diffusion operator $\Delta_{S V V}$, obtained from

$$
\text { (4) } v \cdot \Delta_{s v V}=v \cdot \Delta+\tilde{\Delta}
$$

With $\tilde{\Delta}=\nabla \cdot\left(\varepsilon_{N} Q_{N} \nabla\right)$ and where $\mathrm{N}$ is the degree of the polynomial approximation. The parameter $\varepsilon_{N}$ controls the amplitude of the dissipation (usually $\varepsilon_{N}=O(1 / \mathrm{N})$ ) and $Q_{N}$ represents the SVV-operator kernel. In the Chebyshev or
Fourier space, this operator kernel is defined in such a way that $\hat{Q}_{k}=0$ if $k<m_{N}$ and $1 \geq \hat{Q}_{k} \geq 0$ if $k>m_{N}$. Thus $m_{N}$ is a control parameter which allows selecting the range of frequencies on which the SVV acts (usually $m_{N}=O(\sqrt{N})$ ), refer to Figure 2 . The flow around the bluff body is strongly governed by the boundary layers that develop at the walls, where are located the production and the transfer of turbulence at the smallest scales of the flow. These boundary layers being too thin to be resolved, a near wall treatment has been developed, in the frame of the SVV which initially is a global approach. Here, the near wall treatment (SVV-NW) permits to relax locally the SVV dissipation within the boundary layer through the activation parameter $m_{N}$. To this end, we complete the SVV stabilized Navier-Stokes equations with an additional force term defined as $f_{B L}=\chi_{B L}\left(\tilde{\Delta}_{B L}-\tilde{\Delta}\right) \cdot u$ where $u$ is the velocity and with $\chi_{B L}$, a new characteristic function equal to 1 in the NW region and to 0 elsewhere.

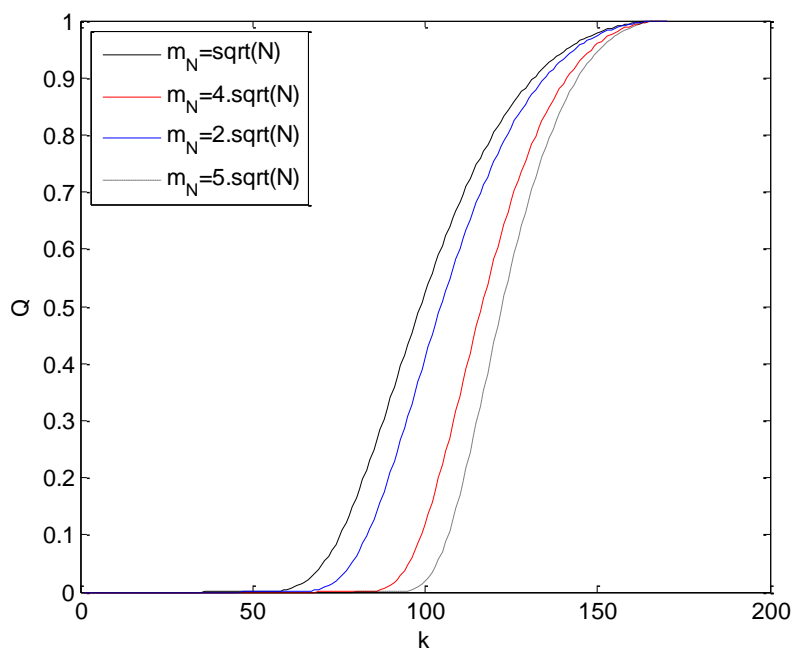

Figure 2: SVV-operator Kernel in the spectral space Sensitivity to the activation parameter $(N=170)$

Simulations have been performed on a NEC SX8 parallelvectorial computer. Statistical steady state has been reached after 6 vortex shedding cycles (i.e Von Karman cycles), that required about $550 \mathrm{CPU}$ hours.

\section{SQUARE CYLYNDER FLOW}

As mentioned for introductory purposes, the square cylinder flow has been regularly studied in the last decades. Several experimental and numerical reference works are available in the literature, (e.g [24], [25], [19], [26], [27]), along with a large amount of results provides a large data base for numerical validation of turbulent flow solver. Before analysing in more details the flow that develops over the square cylinder, it is 
proposed to benchmark averaged quantities obtained by means of the SVV-LES methodology with existing experimental data.

The main relevant quantities for further Flow Induced Vibrations (FIV) analysis are presented in Table 1. The drag and pressure base coefficients predicted by CFD are in close agreement with the experimental values. This excellent agreement clearly assesses the ability of the flow solver to recover the pressure distribution at the cylinder wall, which is well-known to be one of the driven parameters for this class of bluff body flow. A more detailed comparative analysis is achieved in [19] and concludes to the accuracy of the numerical solution predicted by the SVV-LES methodology.

\begin{tabular}{|l|c|c|c|}
\cline { 2 - 4 } \multicolumn{1}{c|}{} & $\mathrm{C}_{\mathrm{D}}$ & $\mathrm{St}$ & $\mathrm{Cp}_{\mathrm{b}}$ \\
\hline Experiments ([27]) & $1.9-2.2$ & 0.132 & \\
\hline Experiments ([26]) & 2.1 & 0.13 & -1.3 \\
\hline SVV-LES & 2.2 & 0.141 & -1.3 \\
\hline
\end{tabular}

Table 1: Mean Quantities Comparison CFD vs. Experiments

The flow that develops over the square cylinder appears to be very complex. On the top and bottom cylinder walls, the flow separates from the cylinder at the leading edge due to the adverse pressure gradient and the sharp geometry. A recirculation forms just behind the separation point and spreads up to the trailing edge. The flow does not reattach on the roof, refer to Figure 3, but in the near wake where a third recirculation zone (of one cylinder diameter length) is formed. Just above and under the bluff body, a shear layer develops at the interface between the low speed of the recirculation and the high speed of the free stream flow. Such shear layer alternatively feeds a spanwise vortex located at the trailing edge level. This stretched vortex grows up in the near wake of the cylinder up to be strong enough and to be advected in the wake of the cylinder. Such scenario, initially described by Gerrard, [28], alternatively happens at the upper and at the lower trailing edge of the cylinder to result in the well-known Von Karman (VK) like Instability. The characteristic dimensionless frequency of such Vortex Shedding in this configuration equal to $S t=0.14$. However, by intermittent means, some VK vortices are less strong that punctually generates load variation (lift and drag). Generally such large scale vortices are characterized by low speed and low pressure zones, refer to Figure 3 and Figure 4. The different sizes of the VK vortices that flow in the wake can be then (at least partially) interpreted as $3 \mathrm{D}$ effects and as vortex strength indicator.

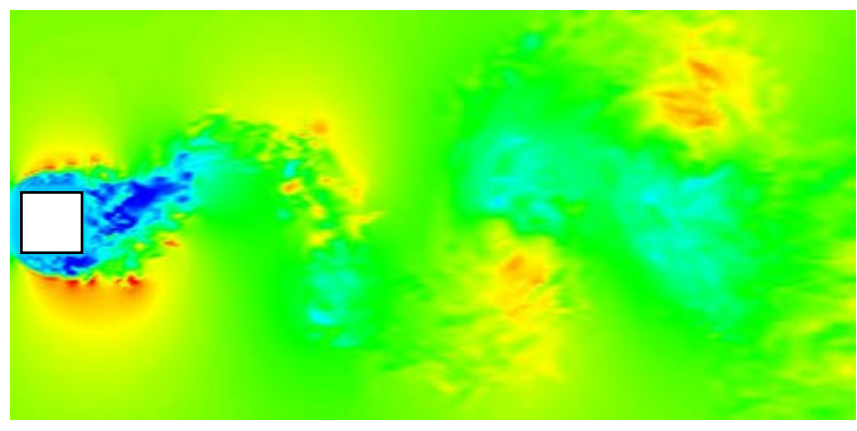

Figure 3: Main Instantaneous Flow Pattern - iso-contours of the stream wise velocity

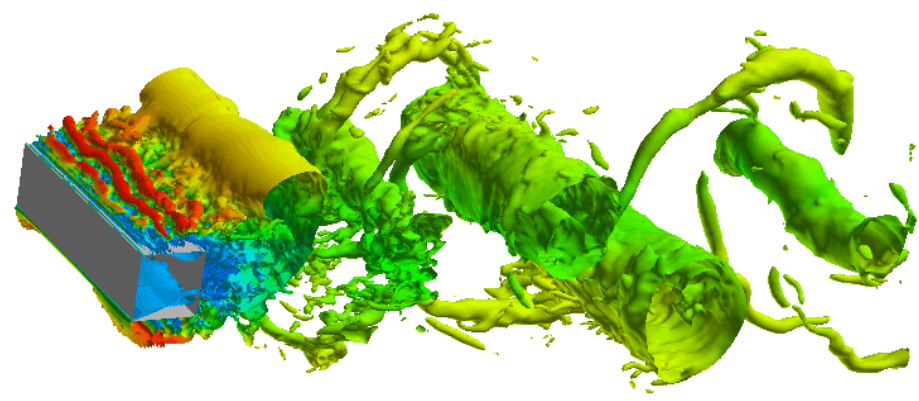

Figure 4: Main Instantaneous Flow Pattern - iso-surface of the pressure fluctuations

As previously observed more upstream, the juxtaposition of the low speed regions (i.e the VK vortices) and of the high speed regions (i.e the free stream current) triggers the development of a shear layer that rolls up over the VK structures, refer to Figure 3. Such shear layer is the place where multi-scale streamwise vortices develop as shown by the iso-surfaces of the pressure fluctuation and of the Q-criterion, refer to Figure 4 and Figure 5.

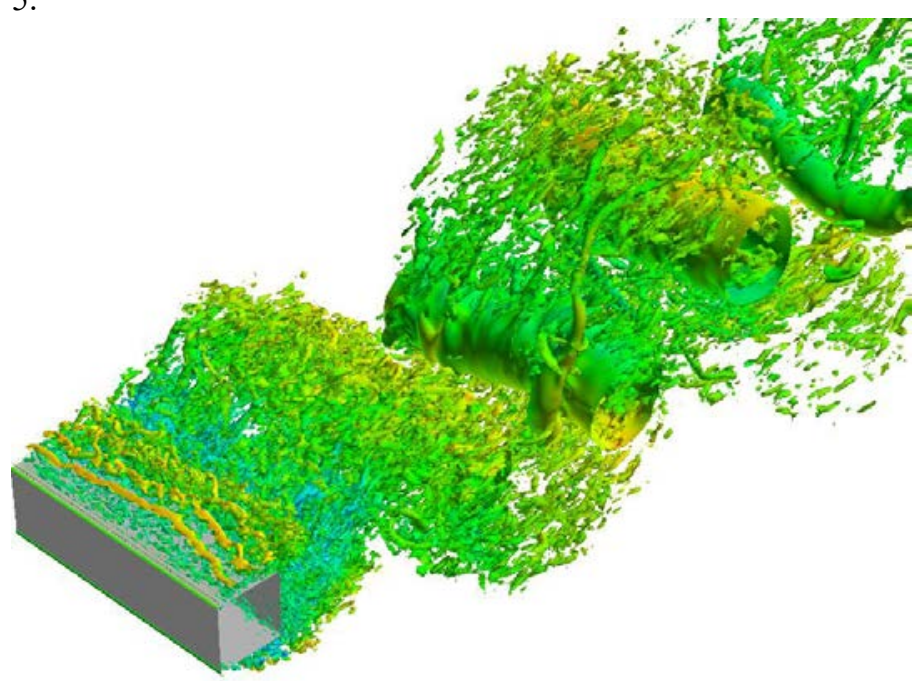

Figure 5: Main Instantaneous Flow Pattern - iso-surface of pressure and of Q-criterion 
Focusing on the vicinity of the cylinder, spanwise vortices form in the shear layers that develop over the upper and lower walls of the bluff body. Such Kelvin Helmholtz (KH) like vorticies are triggered from the leading edge and are advected up to the trailing edge where they are finally absorbed into the VK structures, refer to Figure 4. Such vortical scenario has been already observed and detailed numerically and experimentally in [26] \& [19]. In [26], authors notably pointed out the temporal signature of such $\mathrm{KH}$ vortices formation and underlined the intermittency of such phenomena for different Reynolds numbers. Comparisons between experiments (at $\mathrm{Re}=2000$ ) and numerical visualizations (at $\mathrm{Re}=22000$ ) of the $\mathrm{KH}$ instability demonstrate the ability of the present numeric's to recover such fine dynamical interactions, as shown in Figure 6 . Complementary flow visualization obtained at $\mathrm{Re}=22000$ (and higher) and extracted from [26] confirms that such $\mathrm{KH}$ scenario remains similar at higher $\mathrm{Re}, e . g$ at $\mathrm{Re}=22000$ (Figure 7) and at $\mathrm{Re}=166000$ (refer to [26]). The only noticeable difference is that the size of the KH structures decreases as the Reynolds number increases.
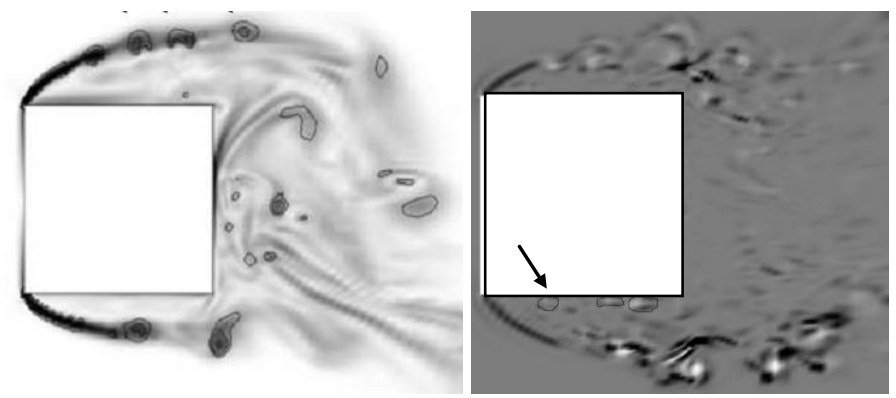

Figure 6: KH Scenario over the Cylinder; Comparison between experiments, [26], at $R e=2000$ (left) and present CFD $R e=2200$ (right); iso-contour of pressure fluctuations

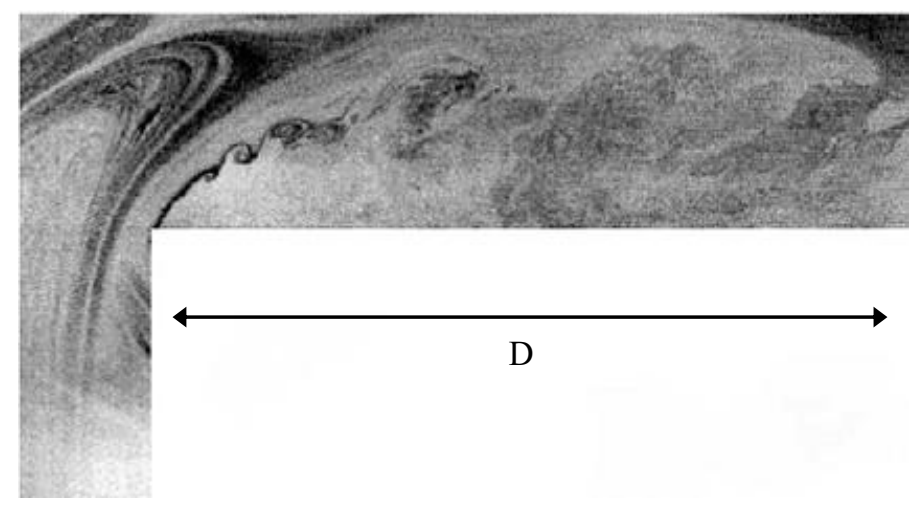

Figure 7: Zoom on the KH Scenario over the Cylinder of diameter D; Results extracted from experiments, [26], at $R e=22000$
Flowing from the leading edge to the trailing edge, $\mathrm{KH}$ vortex grows and slightly lifts. The amplitude of the perturbation equally grows along the shear layer up to reach a maximum at the trailing edge level, as depict by means of the turbulent kinetic energy iso-contours $\left(k=<u u_{i}{ }^{\prime}>2 / 2 / U_{0}{ }^{2}\right)$ plotted in Figure 8. The shear layer consequently appears to be the location of laminar-turbulent transition.

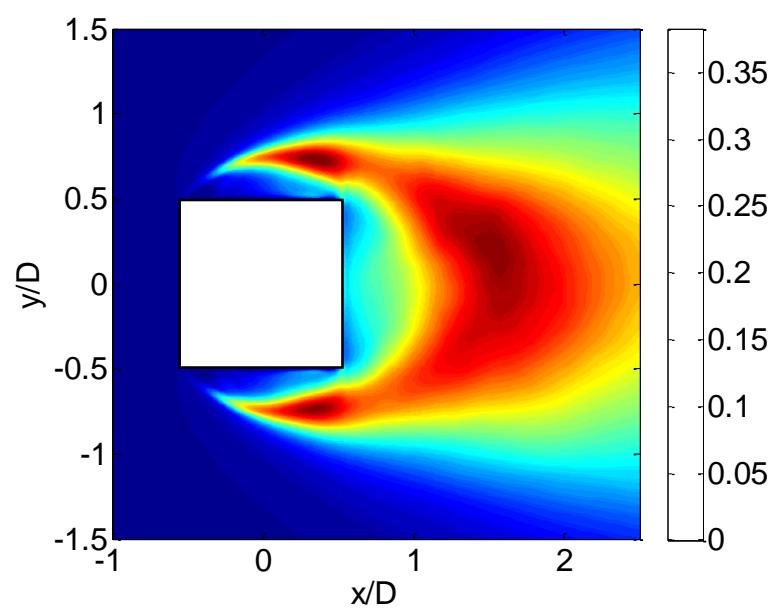

Figure 8: Mean dimensionless Turbulent kinetic energy $k$

At the upper and lower wall vicinity, small vortices flow upriver in the recirculation zone, from the trailing edge up to the leading edge. These vortices are finally absorbed by the shear layer refer to the arrow in Figure 6, which produces an energy feedback in the shear layer. Such process corresponds to the well-known 'back scattering' phenomenon.

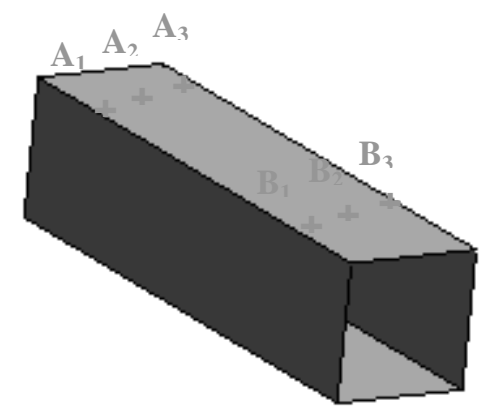

Figure 9: Sketch of the pressure measurement locations

In view of spectral analysis, velocity and pressure have been sampled at different locations in the shear layers. Sampling has been performed for each span wise mesh node but is presented here for two planes only corresponding to $\mathrm{z}=-1.44$ and $\mathrm{z}=1.375$ (denoted respectively planes A \& B). For each of these planes, pressure has been measured at three locations in the shear layer $\left(A_{1} ; x=-0.15 . D\right),\left(A_{2} ; x=0\right), \&\left(A_{3} ; x=0.35 . D\right)$ refer to Figure 9. As already remarked in [26] \& [19], the flow is fully unsteady and intermittent more particularly in the shear layer. Whatever the location at which the pressure was sampled, the spectra underlined the main energetic mode that corresponds to the 
large VK vortices $(S t=0.14)$ developing in the near wake, refer to Figure $10 \&$ Figure 11. Depending on the location in the shear layer (in the stream wise direction), a secondary pick appears at higher frequencies and is related to the $\mathrm{KH}$ vortices $(S t=2.5)$. Depending on the span wise location, such frequency appears more or less far along the cylinder wall and consequently underlines the existence of 3D effects. In plane A, $\mathrm{KH}$ vortices are mainly observable on the second half part of the cylinder wall (from $\mathrm{A}_{2}$ in Figure 10) whereas in plane B, the vortices are generated from the leading edge (from $B_{1}$ Figure 11). Further downstream in the shear layer, at $x=0.35 . D\left(B_{3}\right)$, a first pairing occurs and leads to a broadening of the energy distribution in the low frequency direction. This is a typical backscattering effect observed in the initial transition phase of turbulent shear flows already observed in [26] \& [19]. The resulting energy spectrum underlines a larger band, up to the VK with an intermediary remarkable frequency corresponding to $S t=0.58$. Depending on the span wise location, such pairing may occur or not. This is consistent with this $\mathrm{KH}$ process being observed with a high level of intermittency in [26] and may be correlated to the intermittency inherent to the VK scenario. More investigations are currently in progress whose results will be reported at the time of the conference.

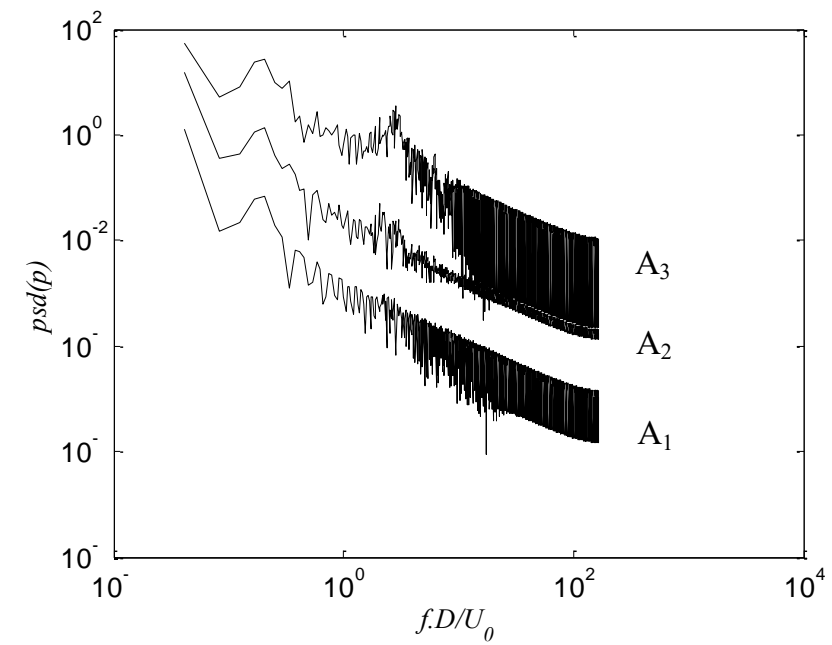

Figure 10: $\mathrm{KH}$ Spectra (pressure) at the three streamwise locations defined in Figure 9 ( $z=-1.4375)$

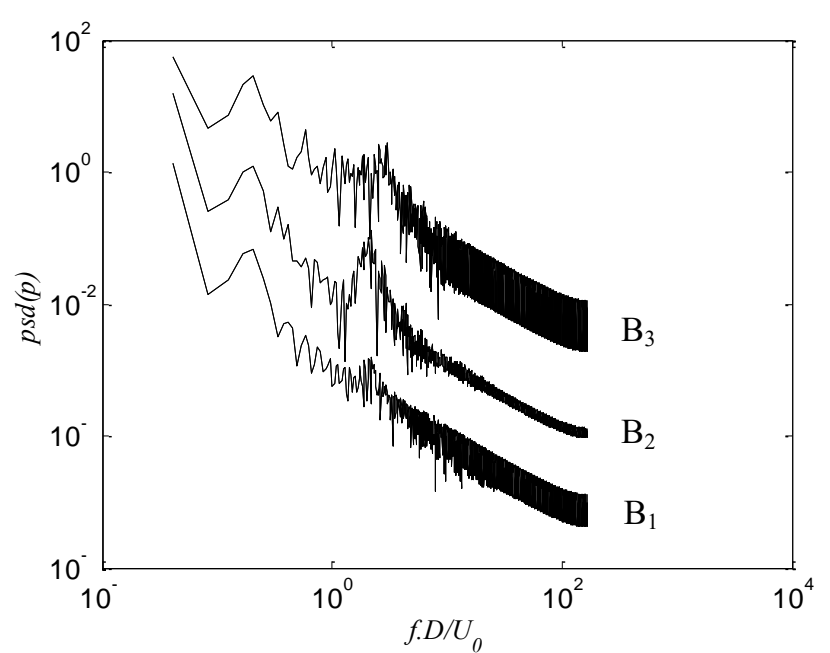

Figure 11: KH Spectra (pressure) at the three streamwise locations defined in Figure 9 ( $z=1.375)$

In view of further FSI investigation, it is fundamental to improve understanding of the role played by such $\mathrm{KH}$ process and its interaction with the VK vortices as well. In such configuration, the energy conveyed by the $\mathrm{KH}$ vortices is strong enough to impact the load acting on the cylinder. The drag spectrum presented in Figure 12 especially points out the existence of a multi frequency loading. As expected, the large scale frequency $(S t=0.14)$ is clearly observable. However, the main frequency $(S t \approx 0.55)$ is related to the $\mathrm{KH}$ vortices that develop in the shear layer over the cylinder, as previously remarked in Figure 11.

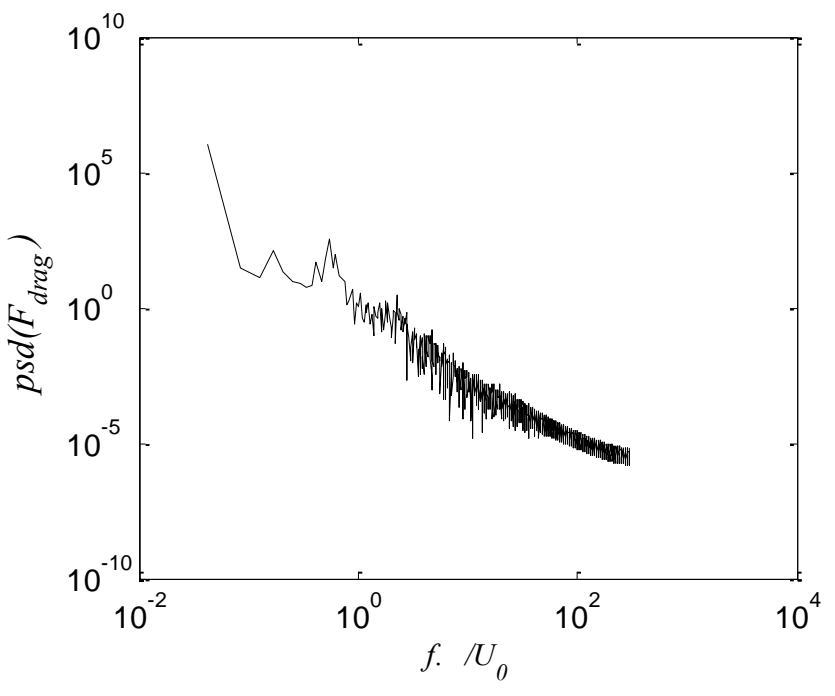

Figure 12: Spectrum of the Drag Force

\section{CONCLUDING REMARKS \& PERSPECTIVES}

The high Reynolds number flow over the square cylinder has been accurately simulated. Comparisons with reference 
works have underlined the ability of this spectral solver to finely recover industrial configuration, with all main hydrodynamic characteristics (loads, frequencies, pressure repartition) being well predicted.

Moreover, the SVV-LES results have underlined the multi-scale nature of the flow developing over such a bluff body. Large scales formed in the near wake of the cylinder are assimilated to VK likes instability that has been temporally signed. Intermittent phenomenon seems however to disturb the VK dynamics. A fine characterization of this process is not yet at hand and should be the subject of further investigation.

More upstream, the shear layer that develops over the cylinder appears as a key location, as it turn to be the location of a transition to a turbulent regime, as well as the location of energy transfer by means of backscattering. The KH vortices generated in this shear layer appear to be strong enough to disturb the VK process intermittently and clearly sign in in the load spectra. The unsteadiness and intermittency of the $\mathrm{KH}$ instability could be consequently one of the main ingredients for the plunge galloping occurrence.

We expect further investigation will allow proposing a consistent characterization of the complex multi scale vortex scenario. However it clearly appears that the shear layer and the resulting $\mathrm{KH}$ instability could be a key location in view of the following wake flow stability \& control analysis.

\section{ACKNOWLEDGMENTS}

The authors would like to thank SEAL Engineering (Technip Group) for encouraging and allowing this paper to be published.

\section{REFERENCES}

[1] Blevins R; D., Flow-Induced Vibration, Second Edition 1990, Van Nostrand ReinHold

[2] C. Berhault, C. Dassibat, J. L. Legras, P. Wiet, A. Cinello, T. Deglaire, B. Molin, Galloping Assessment: Instability Occurrence of Slender Structures of Non Circular Section in current Flow, OTC, 2012, OTC 23113

[3] Dalton C., Xu Y. \& Owen J.C., The suppression of lift on a circular cylinder due to vortex shedding at moderate Reynolds numbers. J. Fluids Struct. 15, 617 (2001).
[4] Illy H., Geffroy P. \& Jacquin L., Control of cavity flow by means of a spanwise cylinder. In Proc. 21st ICTAM conference, Warsaw (2004).

[5] McGrath S.F. \& Shaw L.L., Active control of shallow cavity acoustic resonance. AIAA Paper 96-1949 (1996).

[6] Meliga P., Pujals G. \& Serre E., Sensitivity of 2-D turbulent flow past a D-shaped cylinder using global stability. Phys. Fluids 24:061701 (2012).

[7] Parezanović V. \& Cadot O., Experimental sensitivity analysis of the global properties of a twodimensional turbulent wake. J. Fluid Mech. 693, 115 (2012)

[8] Sakamoto H., Haniu H. \& Tan K., An optimum suppression of fluid forces by controlling a shear layer separated from a square prism. Trans. ASME J. Fluids Eng. 116, 221 (1991)

[9] Strykowski P.J. \& Sreenivasan K.R., On the formation and suppression of vortex shedding at low Reynolds numbers. J. Fluid Mech. 218, 71 (1990)

[10] Oakley O.H. \& Spencer D. 2004 Deepstar VIV experiments with a cylinder at high Reynolds numbers, Proc. DOT Conf., Marseille.

[11] Minguez, M., Luppi, A., Pattedoie, S., Maloberti, R.,'Slender Buoy VIM \& VIR Analysis by CFD/FSI Approach', OMAE2011-49065

[12] Zimmerman, C., James, R., Seguin, B., Lynch, M., Hydrodynamic Properties of an asymmetric Deepwater Riser Bundle, OMAE2006-92469

[13] Sergent, E., De Wilde, J., Harrisson, R., Helle, Y. Vortex Induced Vibration of the GAP Bundle from selection of the Flow Lines Arrangement to VIV Fatigue Analysis, OMAE2008-57538

[14] Tadmor, E., Convergence of spectral methods for nonlinear conservation laws, SIAM, J. of Num, Anal., 26, 30, 1989

[15] Karamanos, G. S. and Karniadakis, G. E., A spectral vanishing viscosity method for large-eddy simulation, J. Comput. Phys. 163, 22, 2000

[16] Pasquetti, R., Spectral Vanishing Viscosity Method for Large-Eddy Simulation of Turbulent Flows, J. Sc. Comput.,27, 365, 2006 
[17] Severac, E., Serre, E., A spectral viscosity LES for the simulation of turbulent flows within rotating cavities, J. Comput. Phys., 226, 1234, 2007

[18] Minguez, M., Pasquetti, R., Serre, E., High-Order Large-Eddy Simulation of the Flow over the 'Ahmed body' Car Model, Phys. of Fluids, 20, 2008

[19] M. Minguez, C. Brun, E. Serre,, Experimental and High order LES analysis of the flow in near-wall region of a square cylinder, Int. J. of Heat and Fluid Flow, 32, 558-566,2011.

[20] Den Hartog, J. P., Mechanical Vibrations, $4^{\text {th }}$ ed., Mc Graw Hill, New York, 1956

[21] Weaver, D. S., Veljkovic, I., Vortex Shedding and Galloping of open semi-circular and parabolic cylinders in cross-flow, J. of Fluids and Struct., 21, 65-74, 2005

[22] Alonso, G., Meseguer, J., Sanz-Andrés, A., Valero, E., On the galloping instability of two-dimensional bodies having elliptical cross-section, J. of Wind Eng. and Ind. Aerodynamics, 98, 2010.

[23] Eça, L., Vaz, G., Workshop on verification and validation of CFD for Offshore Flows, OMAE2012/V\&V-00000.

[24] Rodi, W., Comparison of LES and RANS calculations of the flow around bluff bodies. J. Wind Eng. Ind. Aerodyn., 3-19, 1993

[25] Voke, P., In: 2nd ERCOFTAC Workshop on Direct and Large-Eddy Simulation Grenoble, 1996.

[26] C. Brun, S. Aubrun, T. Goossens, Ph. Ravier, Coherent Structures and their Frequency Signature in the Separated Shear Layer on the Sides of a Square Cylinder, Flow Turbulence Combust, 2008

[27] D.A. Lyn, S. Einav, W. Rodi and J.H. Park, A laser doppler velocimetry study of ensemble-averaged characteristics of the turbulent near wake of a square cylinder, J. Fluid Mech., 304 : 285-319, 1995

[28] J.H. Gerrard, The mechanics of the formation region of vortices behind bluff bodies, J. FluidMech., 25, 401-413, 1966 